Article

\title{
On Identification of Critical Material Attributes for Compression Behaviour of Pharmaceutical Diluent Powders
}

\author{
Jianyi Zhang ${ }^{1}$, Chuan-Yu Wu ${ }^{1, *}$ (D), Xin Pan ${ }^{2}$ and Chuanbin $\mathrm{Wu}^{2}$ \\ 1 Department of Chemical and Process Engineering, University of Surrey, Guildford GU2 7XH, UK; \\ jianyi.zhang@surrey.ac.uk \\ 2 School of Pharmaceutical Sciences, Sun Yat-sen University, Guangzhou 510006, Guangdong, China; \\ mercurypan@foxmail.com (X.P.); chuanbin_wu@126.com (C.W.) \\ * Correspondence: c.y.wu@surrey.ac.uk; Tel.: +44-148-368-3506
}

Received: 6 April 2017; Accepted: 20 July 2017; Published: 23 July 2017

\begin{abstract}
As one of the commonly-used solid dosage forms, pharmaceutical tablets have been widely used to deliver active drugs into the human body, satisfying patient's therapeutic requirements. To manufacture tablets of good quality, diluent powders are generally used in formulation development to increase the bulk of formulations and to bind other inactive ingredients with the active pharmaceutical ingredients (APIs). For formulations of a low API dose, the drug products generally consist of a large fraction of diluent powders. Hence, the attributes of diluents become extremely important and can significantly influence the final product property. Therefore, it is essential to accurately characterise the mechanical properties of the diluents and to thoroughly understand how their mechanical properties affect the manufacturing performance and properties of the final products, which will build a sound scientific basis for formulation design and product development. In this study, a comprehensive evaluation of the mechanical properties of the widely-used pharmaceutical diluent powders, including microcrystalline cellulose (MCC) powders with different grades (i.e., Avicel PH 101, Avicel PH 102, and DG), mannitol SD 100, lactose monohydrate, and dibasic calcium phosphate, were performed. The powder compressibility was assessed with Heckel and Kawakita analyses. The material elastic recovery during decompression and in storage was investigated through monitoring the change in the dimensions of the compressed tablets over time. The powder hygroscopicity was also evaluated to examine the water absorption ability of powders from the surroundings. It was shown that the MCC tablets exhibited continuous volume expansion after ejection, which is believed to be induced by (1) water absorption from the surrounding, and (2) elastic recovery. However, mannitol tablets showed volume expansion immediately after ejection, followed by the material shrinkage in storage. It is anticipated that the expansion was induced by elastic recovery to a limited extent, while the shrinkage was primarily due to the solidification during storage. It was also found that, for all powders considered, the powder compressibility and the elastic recovery depended significantly on the particle breakage tendency: a decrease in the particle breakage tendency led to a slight decrease in the powder compressibility and a significant drop in immediate elastic recovery. This implies that the particle breakage tendency is a critical material attribute in controlling the compression behaviour of pharmaceutical powders.
\end{abstract}

Keywords: pharmaceutical materials; diluent powder; mechanical property; compressibility; particle arrangement; elasticity; hygroscopicity

\section{Introduction}

Pharmaceutical tablets are one of the most preferred dosage forms for drug administration to treat diseases, providing quick therapies to satisfy patient demands [1,2]. During the manufacturing 
process, both active pharmaceutical ingredients (APIs) and inactive excipients play an important role in formulation development. As one of the common inactive excipients, diluent powders are frequently used in the formulation to increase the bulk of tablets and bind all other ingredient components together with the APIs [3]. The selection of diluent powder becomes crucial for product quality control, especially for formulations with a low API dose, where the diluent powder occupies up to $80 \%(w / w)$ of the whole formulation [4].

The influence of the diluent powder property on the tablet quality has been examined by many researchers [5-7]. For instance, Narayan and Hancock [5] studied the relationship between the powder property and the roughness of the tablet surface using diluent powders, such as MCC, mannitol and lactose monohydrate. They concluded that deformation mechanisms affected the mechanical behaviour and surface roughness of the compacts. The tablets made of plastic materials such as MCC were soft, ductile, with high surface variability. On the contrary, mannitol and lactose produced smoother, harder, and more brittle tablets as the main powder consolidation mechanism was particle fragmentation. Akseli et al. [6] investigated the Young's modulus anisotropy of different tablets using the ultrasonic method. They observed that the density of MCC tablets increased more rapidly during compression and more shear deformation took place comparing with lactose tablets. The Young's modulus anisotropy of tablets made of MCC was also more significant than the tablets made of lactose. The compressibility of binary mixtures of ammonio methacrylate copolymers and pharmaceutical diluent powder was investigated by Dave et al. [7]. The particle deformation behaviour of the neat polymers and their mixtures with MCC powder were examined based on the Heckel analysis. The Heckel yield pressure of the polymer-MCC mixture had a good agreement with the values calculated using the mixing rule from the Heckel yield pressure of each pure material and the corresponding component weight fractions.

During tablet manufacturing, mechanical properties of diluent powders, such as compressibility and elasticity, were important as they directly affected the properties of the tablets [5] and of the intermediate products (i.e., granules) [8,9]. In addition, it was also necessary to know the hygroscopicity of the powder because it indicates the sensitivity of the material to the relative humidity. Consequently, this will affect the stability of the products. Furthermore, some problems might occur during the manufacturing process if the properties of the diluent powders are not thoroughly examined. For example, Kottala et al. [10] showed that the interfacial strength of bilayer tablets using the same diluent powder was always stronger than the tablets made of different powders. This is because the usage of the different diluent powders in different tablet layers resulted in elasticity mismatch at the layer interface and significantly increased the delamination risk. Akseli et al. [11] explored the capping tendency of tablets made of different materials and showed that powder properties, such as the effective elastic and the shear moduli, were proved to have impacts on the tablet quality. The lactose tablets always had higher capping tendency compared to MCC tablets produced under the same manufacturing conditions. They concluded that an improper selection of the diluent powders in the formulation might lead to tablet mechanical failures during manufacturing.

Although many previous studies were performed to characterise the mechanical properties of different diluent powders, few of them focused on the relationships between the macroscopic properties (i.e., compressibility, elasticity, and hygroscopicity) and the material microscopic behaviours (i.e., particle movement, particle breakage and particle surface morphology). In addition, there was a lack of understanding of the post compression relaxation behaviours of powders, especially brittle materials (e.g., mannitol powder). Thus, the objectives of this study were to systemically investigate the powder properties of the commonly used pharmaceutical diluent materials, including powder compressibility, particle rearrangement at low-pressure compression, relaxation during decompression, material relaxation in storage, and powder hygroscopicity, and to identify the critical material attributes that dominate the compression behaviour. Such knowledge of material properties and micro-to-macro correlation will guide formulation development and process design to make high quality products. 


\section{Materials and Methods}

\subsection{Materials}

Seven different pharmaceutical diluent powders were considered in this paper, including three microcrystalline cellulose (MCC) powders (FMC, Biopolymer, Philadelphia, PA, USA) with different grades, Avicel PH 102, Avicel PH 101 and DG (i.e., granules were made of a mixture of 75\% w/w MCC and 25\% $w / w$ anhydrous calcium phosphate), mannitol powder (Roquette, Lestrem, France), lactose monohydrate (Foremost, Rothschild, WI, USA), and dibasic calcium phosphate (DCP) (JRS Pharma, Patterson, NY, USA). In addition, magnesium stearate (Avantor Performance Materials, Phillipsburg, NJ, USA) was used as the external lubricant to avoid sticking and capping problems during compression.

\subsection{Tablet Preparation}

A pellet die (Specac Ltd., Orpington, UK) of $13 \mathrm{~mm}$ diameter and a universal material testing machine with a $100 \mathrm{kN}$ load cell (Instron 1175, Norwood, MA, USA) were used to manufacture the tablets. Before compression, a thin layer of magnesium stearate powder was carefully brushed onto the die wall to reduce to the friction between the powder and the die wall during compression. Approximately $700 \mathrm{mg}$ powder was weighed and manually filled into the die. The direct compression method was applied to produce the tablets using flat faced punches. During compression, $60 \mathrm{~s}$ was set to reach maximum loads at a constant compression rate followed by $30 \mathrm{~s}$ for unloading. The tablet ejection speed was controlled at $2 \mathrm{~mm} / \mathrm{s}$. At each experimental condition, three repetitions were performed. The relative humidity and temperature were about $45 \%$ and $21{ }^{\circ} \mathrm{C}$ for all experiments reported here. During compression, the applied loads and the punch movement were automatically recorded using a control system. The compression data was used to plot the stress-strain profile and to assess powder compression behaviour. To produce the tablets with various solid fractions, different compression pressures (i.e., 15, 68, 90, 120, 170, 210, 260, 300, and $340 \mathrm{MPa}$ ) were applied.

\subsection{Compressibility}

During compression, the consolidation of a powder (i.e., decrease of powder bed porosity) was attributed to the intra- and inter-particulate pore space reduction [12]. The powder compressibility, which is defined as the degree to reduce powder volume in a confined space under pressure, indicates the resistance of the powder against the external pressure. Heckel [13] proposed the following equation to analyse the material deformation behaviour:

$$
\ln \left(\frac{1}{\varepsilon}\right)=\mathbf{k P}+\mathbf{A}
$$

where $\varepsilon$ is the apparent tablet porosity, $\mathbf{P}$ is the compression pressure applied. $\mathbf{k}$ and $\mathbf{A}$ are the regression parameters. The tablet porosity was determined by considering the tablet apparent density (calculated using tablet weight and tablet in-die thickness) and the powder true density (determined before compression). In the Heckle plot, $\mathbf{k}$ value was defined as the linear regression slope of the compression stage, and the Heckel yield pressure $\left(\mathbf{P}_{\mathbf{y}}\right)$ was expressed as the reciprocal of the slope. Due to the instability of the yield pressure at low compression pressures, the average Heckel yield pressure was calculated using the in-die compression data with the compression pressure ranging from 200 to $340 \mathrm{MPa}$.

The powder compressibility was also evaluated using the Kawakita equation. The equation described the relationship between the degree of powder volume reduction and the applied pressure as follows:

$$
\frac{\mathbf{P}}{\mathbf{C}}=\frac{\mathbf{P}}{\mathrm{a}}+\frac{\mathbf{1}}{\mathrm{ab}}
$$


The parameter $\mathbf{C}$ (i.e., degree of volume reduction) was calculated by:

$$
\mathrm{C}=\frac{V_{0}-V}{V_{0}}
$$

where parameters $\mathbf{a}$ and $\mathbf{b}$ are constants relating to the total degree of powder volume reduction and the yield strength of particles, respectively. $V_{0}$ and $V$ are the initial and the apparent tablet volumes, respectively. Kawakita parameters, a and $\mathbf{1} / \mathbf{b}$, in Equation (2) were used to characterise powder compressibility using the in-die compression data with the compression pressure from 15 to $340 \mathrm{MPa}$.

\subsection{Particle Rearrangement}

Particle rearrangement dominated the powder consolidation process when a relative low compression pressure was applied. In this case, two possible mechanisms were proposed in the literature to describe particle behaviours: particle movement and particle fragmentation [14]. To distinguish the contribution of these two mechanisms to particle rearrangement, the following equations were used $[15,16]$ :

$$
\begin{gathered}
\rho_{\mathrm{A}}=\mathbf{1}-\mathbf{e}^{-\mathbf{A}} \\
\rho_{\mathrm{B}}=\rho_{\mathrm{A}}-\rho_{0}
\end{gathered}
$$

where $\mathbf{A}$ is the same constant as used in Equation (1). The relative density $\rho_{\mathbf{A}}$ represents the total degree of powder consolidation at the beginning of compression. The relative densities, $\rho_{0}$ and $\rho_{B}$, represent the initial particle rearrangement due to the particle movement and particle fragmentation, respectively. In this study, $\rho_{0}$ was defined as the relative density of the tamped powder bed at a compression pressure of $2 \mathrm{MPa}$.

\subsection{Elasitic Recovery}

The elastic recovery of pharmaceutical powders was investigated during decompression and in storage. In this study, MCC PH 102 and mannitol powders were selected as the model materials. The immediate elastic recovery $\left(\mathbf{V}_{\text {IER }}\right)$ (i.e., in-die elastic recovery) was used to estimate the material elastic recovery occurred during decompression, considering the fast elastic recovery and a part of viscoelastic recovery (i.e., time-depended recovery). The tablet dimensions in the decompression process were recorded using the control system and Equation (6) was used to calculate $\mathbf{V}_{\text {IER }}$ [17]:

$$
\mathrm{V}_{\text {IER }}(\%)=\frac{\mathrm{V}_{\mathrm{f}}-\mathrm{V}_{\text {min }}}{\mathrm{V}_{\text {min }}} \times \mathbf{1 0 0}
$$

where $\mathbf{V}_{\mathbf{f}}$ is the tablet volume at the end of the decompression, $\mathbf{V}_{\min }$ is the tablet volume at the maximum compression pressure.

The compressed tablets continuously exhibited material elastic recovery after ejection. The short-term elastic recovery $\left(\mathbf{V}_{\text {SER }}\right)$ (i.e., out-of-die elastic recovery up to eight hours after ejection) was used to determine the time-dependent relaxations considering the dimension change in both radial and axial directions. Tablet diameter and thickness were measured using a micrometer (Mitutoyo, 293-340, Andover, UK) with an accuracy of $0.0001 \mathrm{~mm}$ at different time instants after ejection: 2, 5, 10, $20,40,60,120,210,300$, and $480 \mathrm{~min}$. The average values were calculated based on three repetitions to minimise the measurement error. All the measurements were taken at the ambient condition. The short-term elastic recovery was obtained using the following equation [18]:

$$
V_{S E R}(\%)=\frac{V-V_{e j}}{V_{e j}} \times 100
$$

where $V$ is the tablet volume at a given time instant during storage, and $V_{e j}$ is the tablet volume at the end of the ejection process. 


\subsection{Hygroscopicity}

The powder was conditioned in an environmental chamber (Climacell, MMM group, München, Germany) to control its water content. The powder was evenly spread on a flat tray and kept in the environmental chamber for at least $24 \mathrm{~h}$. The relative humidity in the chamber was set at $10 \%, 25 \%$, $40 \%, 55 \%, 70 \%$, and $85 \%$. The prepared powder was then removed to a moisture analyser (Ohaus, MN35, Parsippany, NJ, USA) to measure the water content. The mass loss on drying method was applied and the powder was heated at $110^{\circ} \mathrm{C}$ for $5 \mathrm{~min}$ to ensure all water was evaporated. The water content was then calculated as follows:

$$
\varphi_{\mathrm{wc}}(\%)=\frac{\mathbf{m}_{\mathrm{w}}}{\mathbf{m}_{\mathrm{t}}} \times 100
$$

where $\mathbf{m}_{\mathbf{w}}$ is the mass of the water and $\mathbf{m}_{\mathbf{t}}$ is the total mass of the powder before the heat treatment.

\section{Results and Discussion}

\subsection{Compressibility}

In this study, Heckel analysis was performed to explore the material behaviour of different powders. According to the literature, the Heckel yield pressure $\left(\mathbf{P}_{\mathbf{y}}\right)$ was commonly used to describe powder compressibility as it was directly related to powder plastic behaviour $[19,20]$ and the hardness of particles [21]. The larger the Heckel yield pressure, the greater the effort needed to achieve the specific solid fraction during powder compression. The Heckel yield pressures of different pharmaceutical diluent powders at different compression pressures (from 15 to $340 \mathrm{MPa}$ ) are shown in Figure 1. It is shown that the Heckel yield pressure increases with the increasing compression pressure if the compression is relatively weak. However, the Heckel yield pressure reaches a plateau when the compression pressures are higher than $200 \mathrm{MPa}$. This trend is in good agreement with the experimental results published by Patel et al. [22], who examined several pharmaceutical materials including the MCC powder. The authors addressed the material elastic deformation and strain hardening as the main reasons causing the increase in the Heckel yield pressure. In order to characterise the powder property using the Heckel analysis, the arithmetic average Heckel yield pressure was calculated, considering the Heckel yield pressures obtained (i.e., data points at the right side of dashed line in Figure 1) at compression pressures from 200 to 340 MPa. MCC PH 101 and MCC PH 102 powders had similar Heckel yield pressures, which were smaller than that of other powders. Dibasic calcium phosphate (DCP) had the highest Heckel yield pressure, indicating the powder bed was more difficult to consolidate during compression. The addition of DCP to MCC powder makes MCC DG powder with an increased Heckel yield pressure compared with pure MCC powders. Mannitol and lactose powders had medium Heckel yield pressures, which were higher than MCC powders but lower than the DCP powder.

It has been reported that the MCC powders are classified as the plastic materials [22-24], but mannitol [21,25], lactose [26,27], and DCP [22,28] powders are treated as brittle materials. The different predominant consolidation mechanisms were used to explain the Heckel yield pressure variations for different powders [29]. During the compression of plastic powders, the volume reduction process was predominately controlled by particle deformation. According to Heckel analysis, the plastic powders, such as MCC PH 101 and MCC PH 102, had small Heckel yield pressures in comparison with other materials (see Figure 2), indicating a good compressibility of powders. On the other hand, brittle materials showed larger Heckel yield pressures and the powders were more difficult to be compressed compared with plastic powders. The particles of brittle materials tended to break into small fragments during compression. During the particle fracture process, a large amount of energy was needed to break larger particles and create new particle surfaces. This makes brittle materials more difficult to be consolidated. Among all the tested powders, DCP showed the worst compressibility as it had the largest Heckel yield pressure. Comparing with MCC PH 101 and 102, 
MCC DG had a reduced compressibility due to the existence of DCP in its formulation. The results were consistent with the observations published by other researchers [16,30], where lactose and DCP powders were characterised as the brittle materials and their particle plastic deform feature was less than MCC powders.

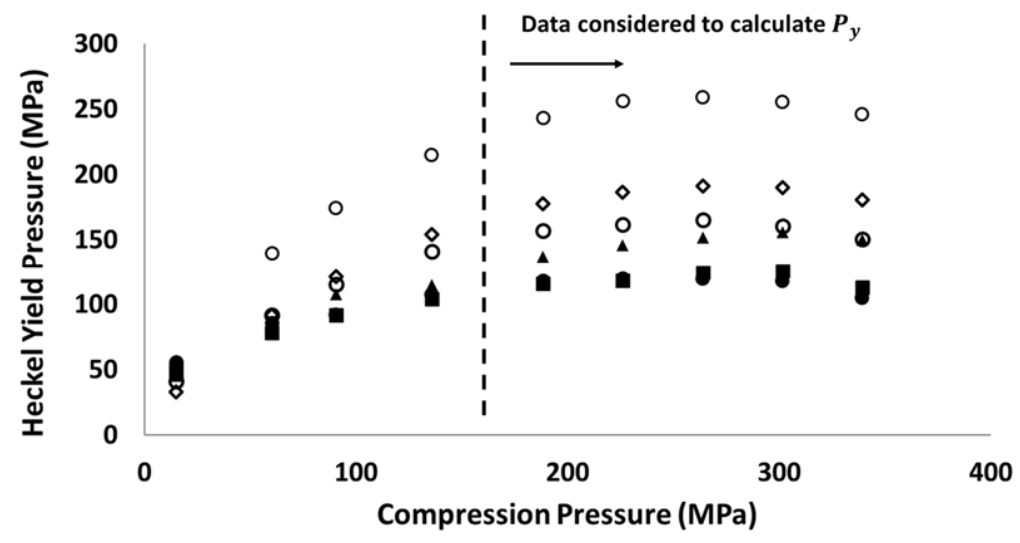

Figure 1. Heckel yield pressure as a function of compression pressure for different diluent powders:

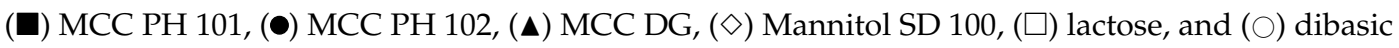
calcium phosphate.

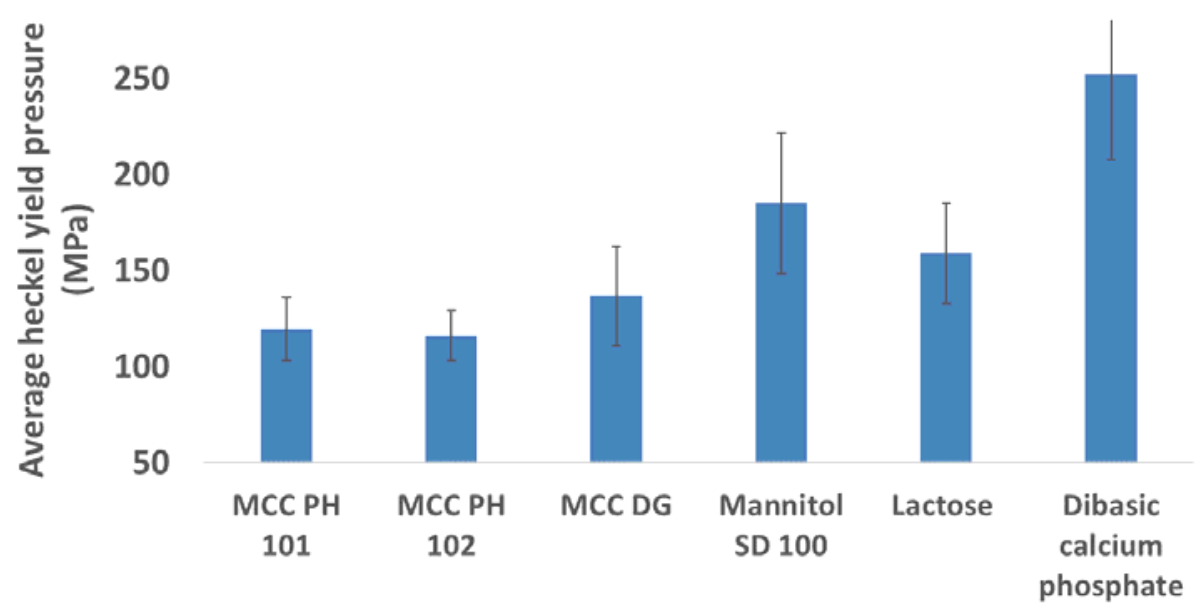

Figure 2. The average Heckel yield pressures for different diluent powders.

The parameters $\mathbf{a}$ and $\mathbf{1 / b}$ in the Kawakita equation (Equation (2)) were also used to evaluate the powder behaviours, as they represented the degree of the compression (i.e., engineering strain) of the powder at the infinite pressure and the compression effort needed to consolidate the powders to achieve half of its total engineering strain, respectively [31]. It was shown that the parameter a was independent of the compression pressure, but parameter $1 / \mathbf{b}$ gradually increased with the increase of compression pressure. The arithmetic means of Kawakita parameters a and $\mathbf{1} / \mathbf{b}$ were used to characterise the property of the materials, as shown in Figure 3, considering three repetitions at each compression pressure. It is clear from Figure 3 a that MCC powders had a higher value of parameter a comparing to other materials, such as mannitol and lactose, indicating that MCC powders could be consolidated more significantly than brittle materials (i.e., the experienced more volume reduction) at the infinite compression pressure. The dominant consolidation mechanism of MCC powders was particle deformation. On the other hand, the volume reduction of brittle materials was mainly due to the fragmentation of particles, and the contribution of particle deformation to powder consolidation 
was very limited. The analysis of parameter a indicated that plastic materials had better compressibility than brittle materials, which was in good agreement with the Heckel analysis.

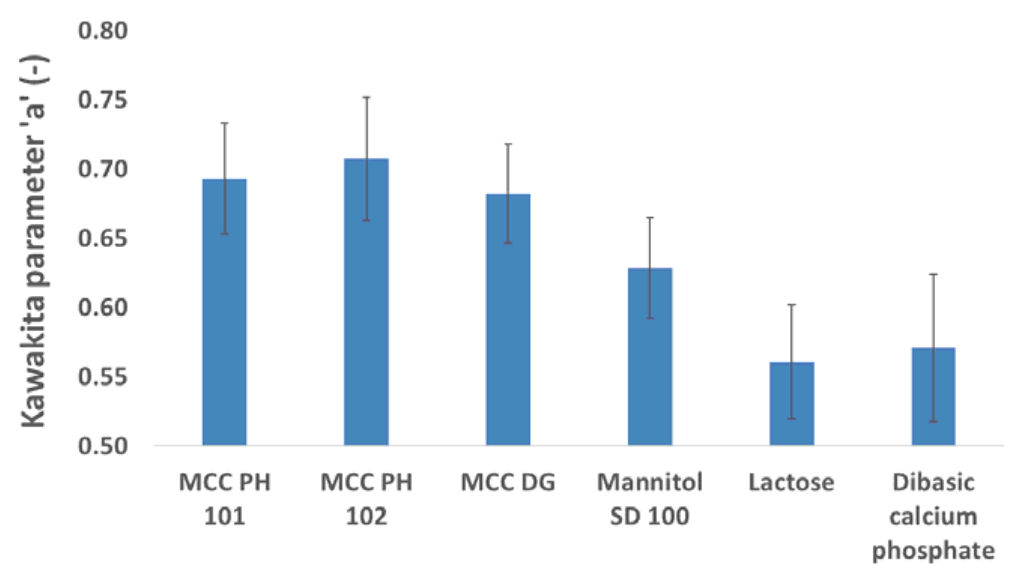

(a)

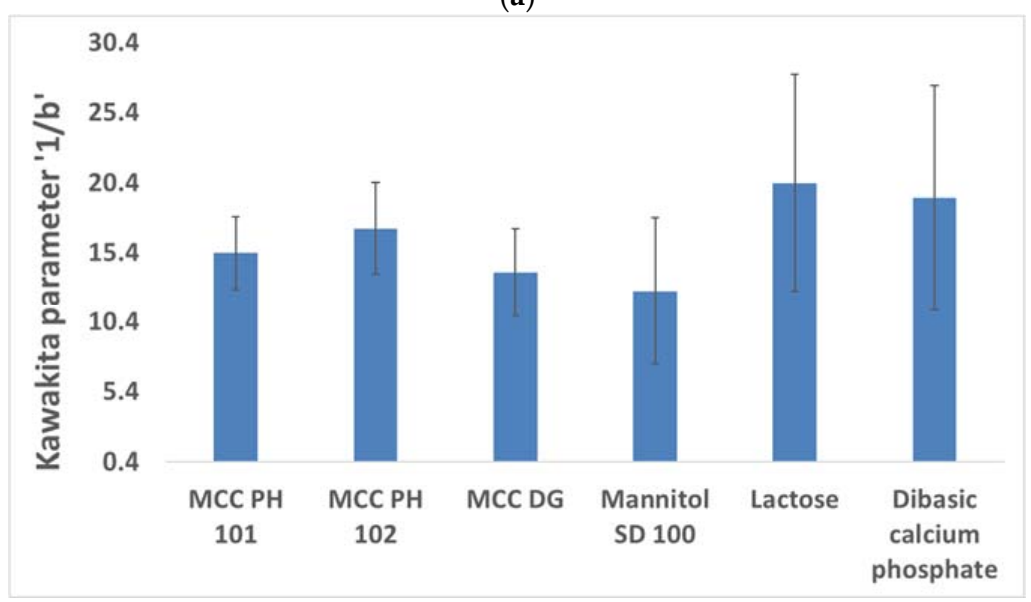

(b)

Figure 3. The Kawakita parameters $\mathbf{a}$ and $\mathbf{1} / \mathbf{b}$ for different diluent powders.

The average value of the Kawakita parameter $\mathbf{1} / \mathbf{b}$ for different powders were shown in Figure $3 \mathrm{~b}$. According to its definition, the parameter $\mathbf{1} / \mathbf{b}$ was related to the compression effort used to achieve half of the maximal theoretical engineering strain. The plastic materials (e.g., MCC powders) were easier to be compressed as they have smaller values for parameter $\mathbf{1} / \mathbf{b}$ compared to brittle materials (e.g., lactose and DCP powders). In the Heckel analysis, the compressibility of mannitol powder was worse than MCC powders, but this conclusion was not consistent with the Kawakita analysis as mannitol had a similar value for parameter a and $\mathbf{1} / \mathbf{b}$ to MCC powders. This abnormal observation could be explained by considering the particle strength of the mannitol powder. The individual mannitol particle was weaker than the particle of lactose or DCP powder, hence, less compression effort was needed to crush the mannitol particle. This hypothesis was proved by analysing particle behaviours in Section 3.2 as more significance of the particle breakage was found for the mannitol powder than any other powders. The error bars shown in Figure $3 \mathrm{~b}$ were due to the strain hardening phenomenon during the compression.

\subsection{Particle Rearrangement}

In the Heckel analysis, the relative density $\rho_{\mathrm{A}}$ was calculated using Equation (4), and used to characterise the overall particle behaviour in compression at small compression pressures (i.e., initial stage of the compression). As described in Equation (5), the relative densities $\rho_{0}$ and $\rho_{B}$ were used 
to characterise the tablet density increase due to the particle position movement and the particle fragmentation, respectively. The overall particle rearrangement for different powders were shown in Figure 4. The MCC powders, such as MCC PH 101 and PH 102 powder, had smaller $\rho_{\mathrm{A}}$ than other powders, indicating a limited particle movement during compression. But the analysis of particle rearrangement depending on just the relative density $\rho_{\mathrm{A}}$ was not perfect because both particle movement and particle fragmentation contributed to the overall particle behaviour. Further analysis was needed to distinguish the dominate densification mechanism for a specific powder.

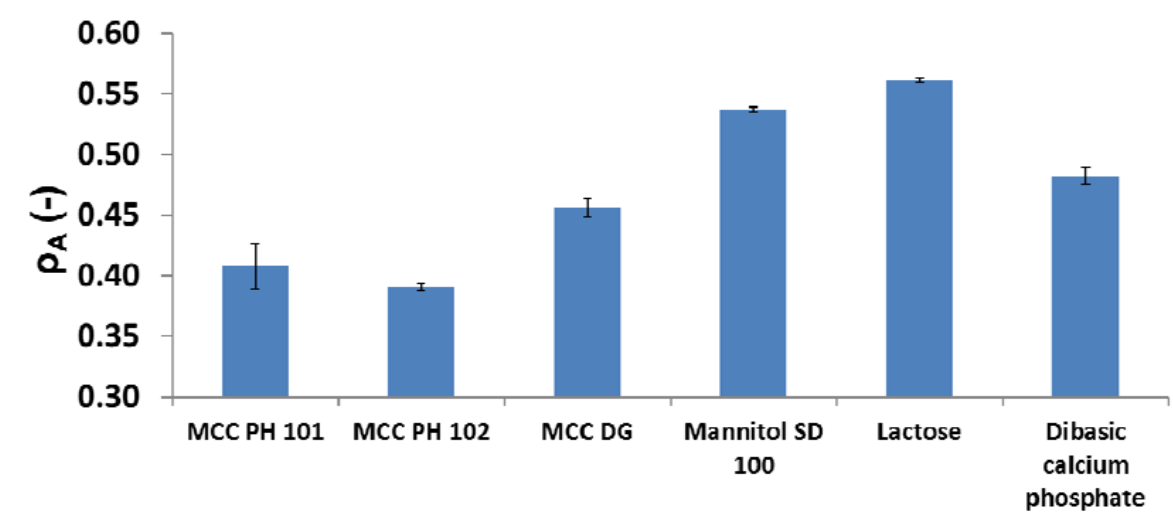

Figure 4. The relative density $\rho_{\mathrm{A}}$ for different diluent powders.

Patel et al. [32] reported that the parameter $\rho_{0}$ was relevant to the magnitude of the particle relocation and could be measured during the compression at low compression pressures. In this study, $\rho_{0}$ was equal to the apparent solid fraction of the powder bed at the compression pressure of $15 \mathrm{MPa}$ (i.e., $100 \mathrm{~N}$ was applied). Under this circumstance, only particle movement (i.e., powder flow) was assumed to contribute to the increase in the powder bed solid fraction because the compression pressure was too small to break the particles. The relative density $\rho_{0}$ for different powders was shown in Figure 5. MCC powders had a smaller $\rho_{0}$ value comparing to other materials. The limited particle movement of MCC powders may be explained by a hypothesis considering the particle shape and particle surface roughness. MCC PH 102 and mannitol SD 100 powders were selected to demonstrate the analysis. The SEM images of these two materials are shown in Figure 6. The particle shape of MCC PH 102 was non-spherical (i.e., needle shape) and the particle surface was rougher compared with the mannitol particles. The non-spherical shape and rough particle surface increased the particle-particle friction and made particles difficult to adjust their position by particle movement.

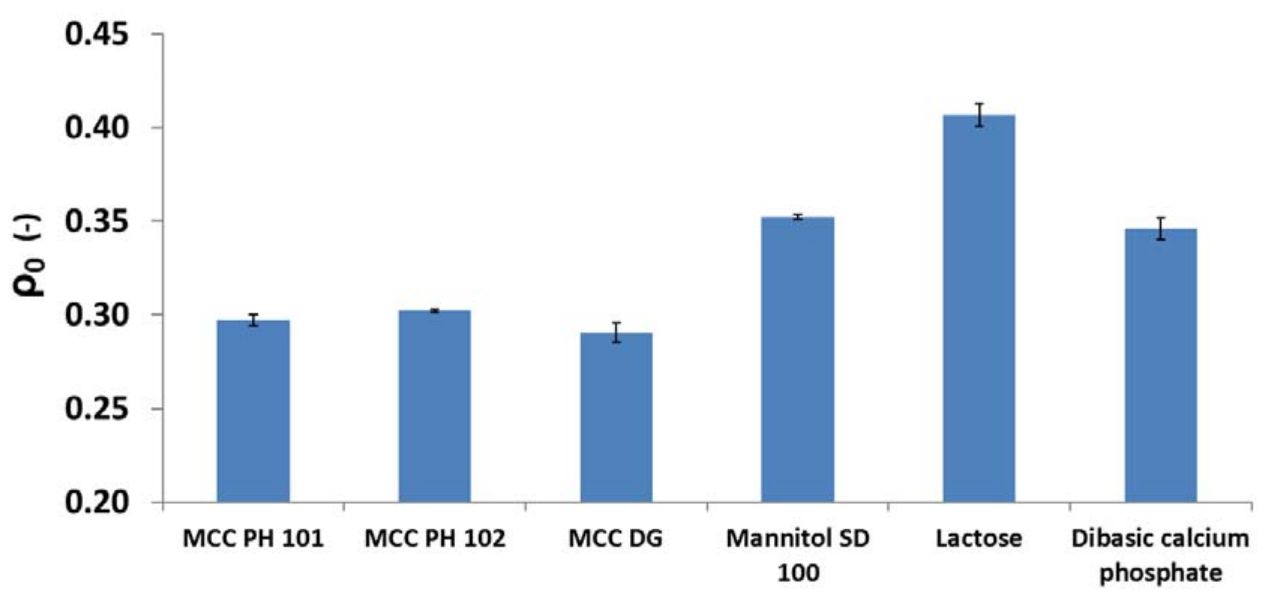

Figure 5. The values of parameter $\rho_{0}$ (i.e. the significance of particle rearrangement) for different diluent powders. 


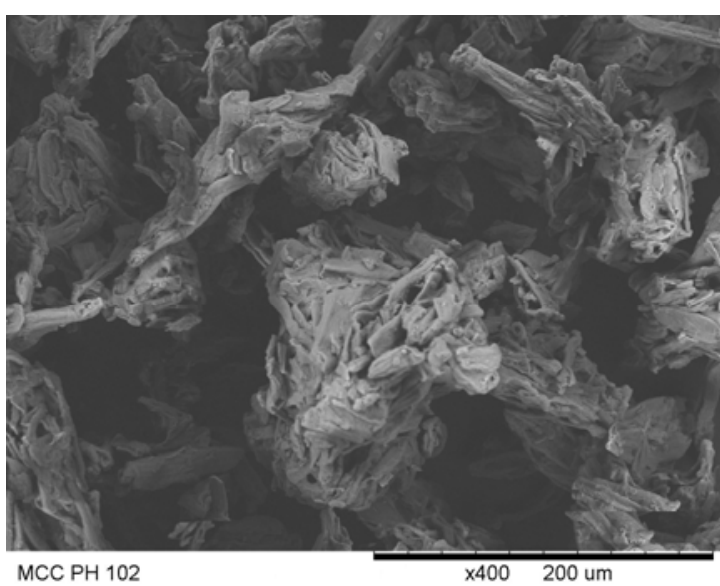

(a)

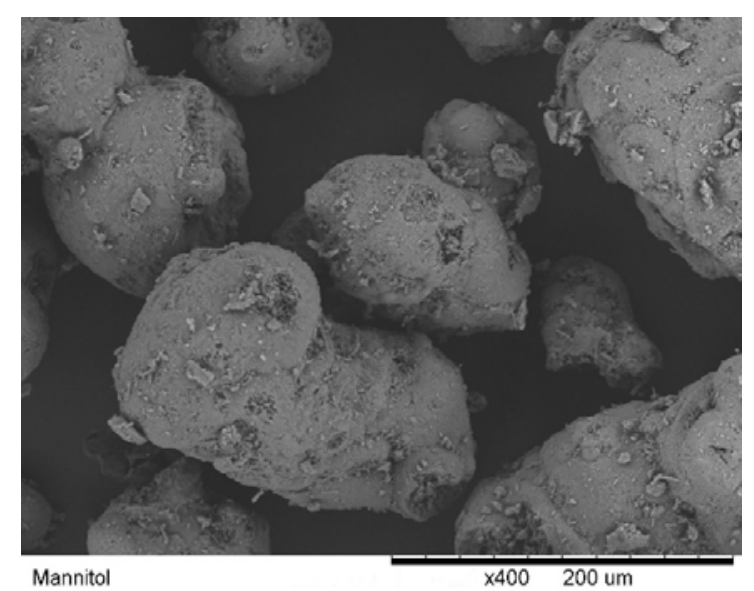

(b)

Figure 6. SEM images of MCC PH 102 (a) and mannitol SD 100 (b) [33].

According to Equation (5), the contribution of particle fragmentation to the powder consolidation was examined using the relative density $\rho_{\mathrm{B}}$. Figure 7 listed all the $\rho_{\mathrm{B}}$ values for the powders tested. Based on the discussion in Section 3.1, plastic materials had relatively small $\rho_{B}$ compared to brittle materials as the particles experienced more particle deformation rather than particle fragmentation during compression. The experimental results supported the material classification: the contribution of particle fragmentation to the tablet density of MCC PH 101 and MCC PH 102 powders were smaller than the brittle materials, such as mannitol, lactose, and DCP powders. This confirmed that the brittle materials experienced significant particle fragmentation in the compaction cycle. Moreover, the density $\rho_{\mathbf{B}}$ for different brittle powders were not similar. Mannitol powder clearly had a larger $\rho_{\mathbf{B}}$ value than the other two powders, indicating that more particle breakage occurred during compression. To explain this phenomenon, a hypothesis was proposed considering the impact of the individual particle strength on the significance of overall powder fragmentation. The individual particle strength of lactose or DCP powders was stronger in comparison with mannitol powder, this allowed more lactose and DCP particles to survive from the breakage at the initial compression. Govedarica et al. [34] examined the single-particle strength of different pharmaceutical powders, including MCC PH 102 and lactose monohydrate. They suggested that single-particle strength greatly affected the powder's predominant consolidation mechanism, which supports the proposed hypothesis.

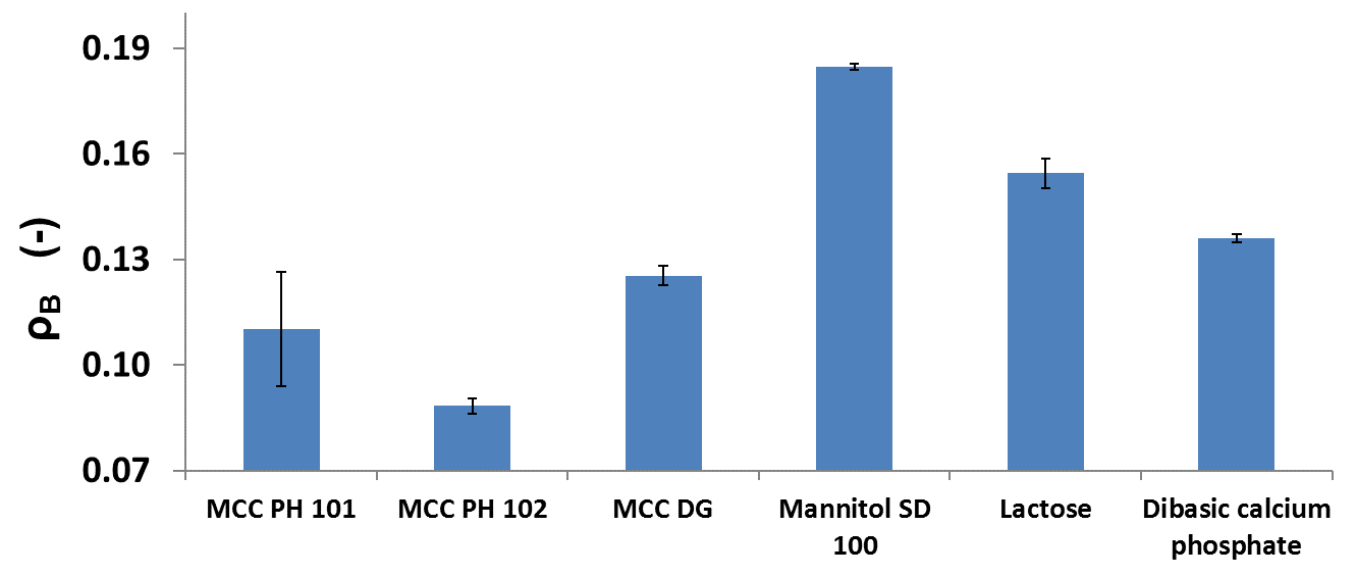

Figure 7. The parameter $\rho_{\mathrm{B}}$ for different powders, indicating the significance of particle fragmentation for different diluent powders. 


\subsection{Elastic Recovery}

The 'in-die elastic recovery' was investigated to determine the powder expansion behaviour during decompression. It was found that the immediate elastic recovery (IER) was not affected by the applied compression pressure, so the arithmetic average of IER at different compression pressures (i.e., nine different measuring points were considered from 15 to $340 \mathrm{MPa}$ ) was calculated to characterise the powder fast recovery (see Figure 8). The tested materials fell into two categories as MCC PH 101 and MCC PH 102 powders had obviously larger IERs than other materials, such as mannitol, lactose, and DCP powder. This is consistent with the particle deformation behaviour during compression, as shown in Figure 3, i.e., the powders experiencing large particle deformation also had larger IERs during the decompression process. When the powder particle exhibited more deformation under pressure, it also had a better chance to store more elastic energy and recovered more volume after the compression pressure was removed [35,36]. Celik and Travers [37] also determined the immediate elastic recovery of some pharmaceutical excipients. For MCC PH 101 and DCP powder, the IER values were $3.5 \%$ and $2 \%$, which were similar to the values obtained in this study.

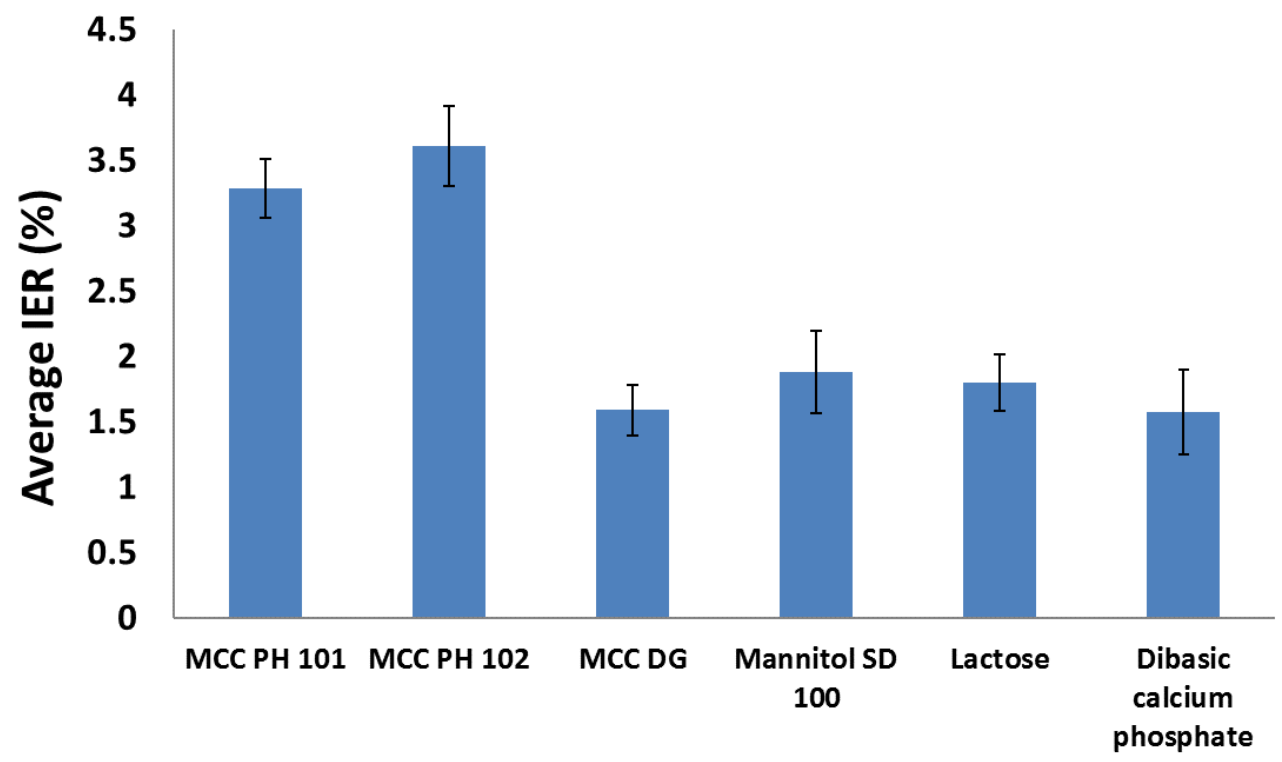

Figure 8. The average IERs for the different materials investigated.

As discussed in the literature [14,36], the volume of the tablet continued to change for a long time after the manufacturing process. It is important to study the material behaviours during storage for both plastic and brittle materials. In this study, MCC PH 102 and mannitol SD 100 powder were selected as the model materials. The tablet volume variations were investigated considering the dimension change in both axial and radial directions (i.e., the thickness and diameter characterizations). In Figure 9a, the accumulated elastic recovery of MCC PH 102 tablet was monitored at different time points in storage. It was noticed that most of tablet volume expansion occurred along the axial direction. The increase in tablet diameter was less than $0.5 \%$ in $8 \mathrm{~h}$ after ejection. This was attributed to the low Poisson's ratio of MCC powders [38]. For all tablets compressed with different pressures, the material exhibited volume expansion in the first $8 \mathrm{~h}$ of the storage. The expansion rate was relatively large in the first hour after the ejection and slowed down as the tablets were stored for a longer time. To characterise the material relaxation behaviour in storage, the average SER was calculated from the accumulated tablet volume change after $100 \mathrm{~min}$ in storage and presented in Figure $9 \mathrm{~b}$. The MCC tablets compressed with low pressures recovered more comparing to the one produced at higher compression pressures. This is because the compression at high pressures (e.g., 210 or $300 \mathrm{MPa}$ ) enabled the stronger particulate interactions by forcing all particles together, creating the denser tablet 
structure. The intensive particle-particle attraction force obstructed the material expansion in the relaxation process which, consequently, led to a reduced SER for the highly-compacted tablets.

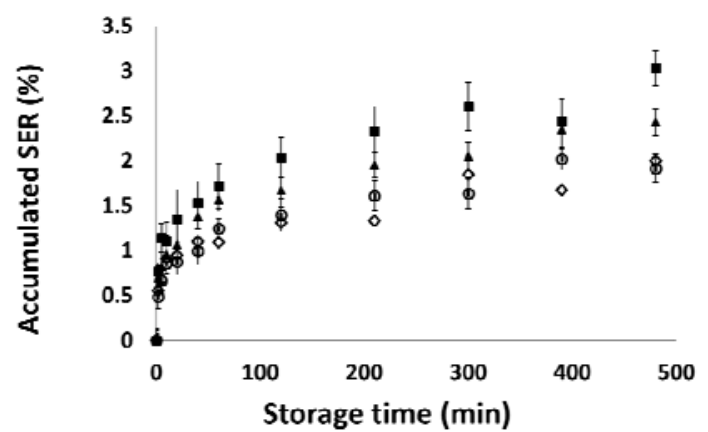

(a)

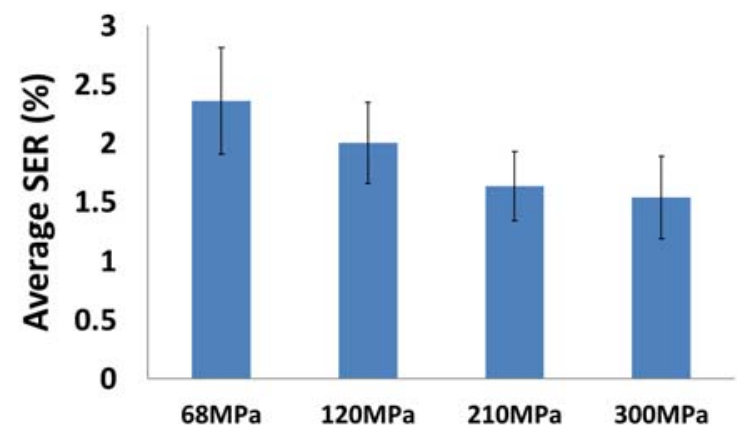

(b)

Figure 9. (a) The accumulated volume changes of MCC PH 102 tablets compressed at different pressures

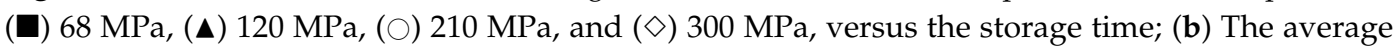
volume expansion at different compression pressures.

The relaxation behaviour of mannitol powder in storage was also examined and the compressed tablet volume changes are shown in Figure 10. The mannitol powder firstly exhibited the similar volume expansion behaviour as MCC PH 102 powder within about $30 \mathrm{~min}$ after ejection. However, the expansion behaviour did not last long. Instead, it is interesting to observe that the tablet experienced volume reduction after $30 \mathrm{~min}$ in storage, which continued in the measurement period considered here (i.e., $8 \mathrm{~h}$ after ejection). The tablet volume became smaller than that at ejection after about two and half hours in storage. Mannitol powder was characterised as a brittle material and the particles did not deform much during compression. As expected for brittle materials, the total volume changes of mannitol tablets (i.e., less than $0.4 \%$ ) was smaller than the MCC tablets (i.e., about $2 \%$ ). Furthermore, the SER did not show any strong dependency on the compression pressure and the tablet shrinkage behaviour was observed for all mannitol tablets compressed at different pressures.

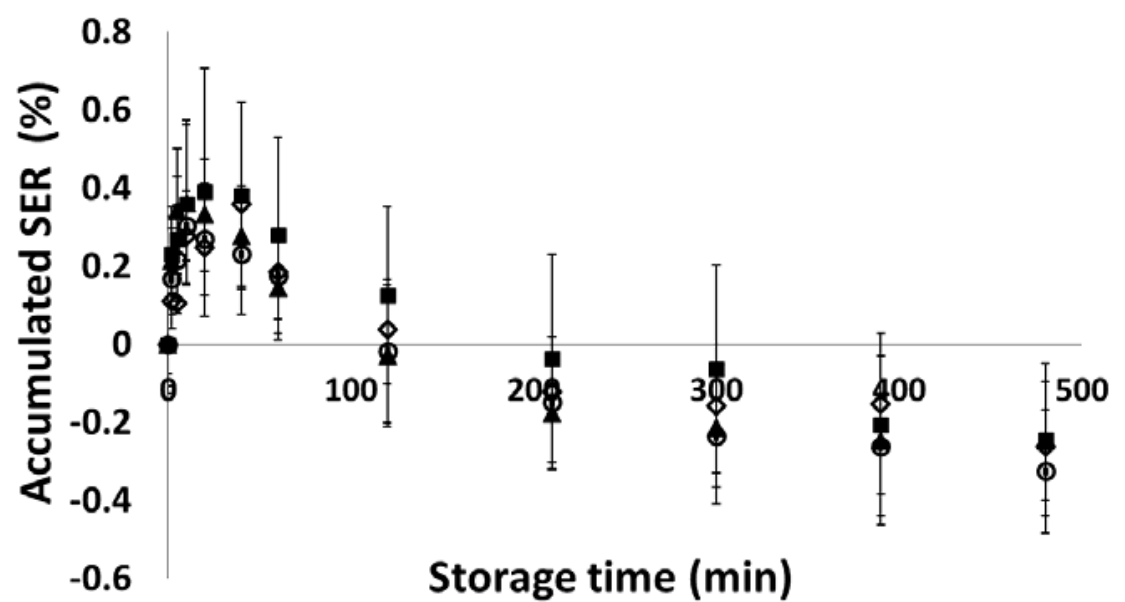

Figure 10. The accumulated volume changes of mannitol tablets compressed at different pressures $68 \mathrm{MPa},(\boldsymbol{\Delta}) 120 \mathrm{MPa},(\bigcirc) 210 \mathrm{MPa}$, and $(\diamond) 300 \mathrm{MPa}$.

\subsection{Hygroscopicity}

In order to better understand the elastic recovery during storage, hygroscopicity of all powders considered in this study was measured and presented in Figure 11. It was clear that the tested materials could be classified into two categories: MCC powders could absorb more water than other 
powders, and the water content increased with the environmental humidity increase. On the other hand, mannitol, lactose, and DCP powders exhibited the similar water absorption ability and the water content in these powders was stable $(\sim 2 \%, w / w)$ and were independent of the tested environmental humidity. Since the MCC DG powder had some dibasic calcium phosphate, the water content for the MCC DG powder showed a similar trend to that of MCC powders, but less water was absorbed. The better hygroscopicity of MCC powders was due to particle surface roughness. As shown in Figure 6, the MCC particles had rough surface, providing large area for water molecules to attach. Furthermore, different types of the crystals were also reported as a factor affecting material hygroscopicity [39]. It is, hence, expected that the expansion of MCC tablets during storage (see Figure 9a) would be more significant when the tablets were stored in high relative humidity condition, as a significant amount of water can be absorbed due to its high hygroscopicity.

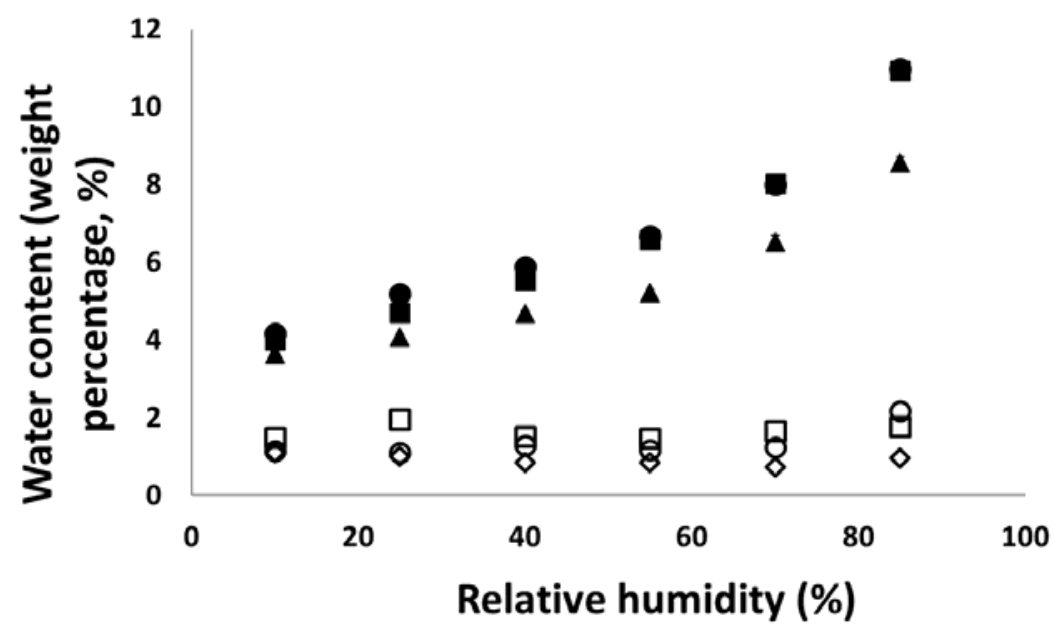

Figure 11. The water contents of different powders stored at different environmental humidity. Key:

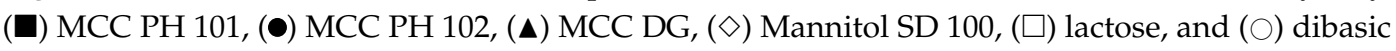
calcium phosphate.

\subsection{Relationships between Particle Attributes and Powder Property}

The relationship between the material compressibility and the significance of the particle breakage was shown in Figure 12. As discussed previously, the relative density from the Heckel analysis was related to particle breakage during compression. Instead of using the Heckel yield pressure, Kawakita parameter a was selected to characterise the powder compressibility. For the diluent powders considered, the material compressibility had a negative relationship with the significance of the particle breakage during compression, which means that the particles of the powder with a good compressibility tended to show a lesser degree of particle fragmentation. It is clear that MCC powders had a better compressibility and exhibited less particle breakage under pressure (i.e., large Kawakita parameter a and small $\rho_{\mathbf{b}}$ ). On the other hand, particles of mannitol, lactose, and DCP powders were fragmented into smaller pieces during the manufacturing process (i.e., small Kawakita parameter a and large $\rho_{\mathrm{b}}$ ). A higher compression pressure was required to facilitate particle breakage, which made these materials less compressible. 


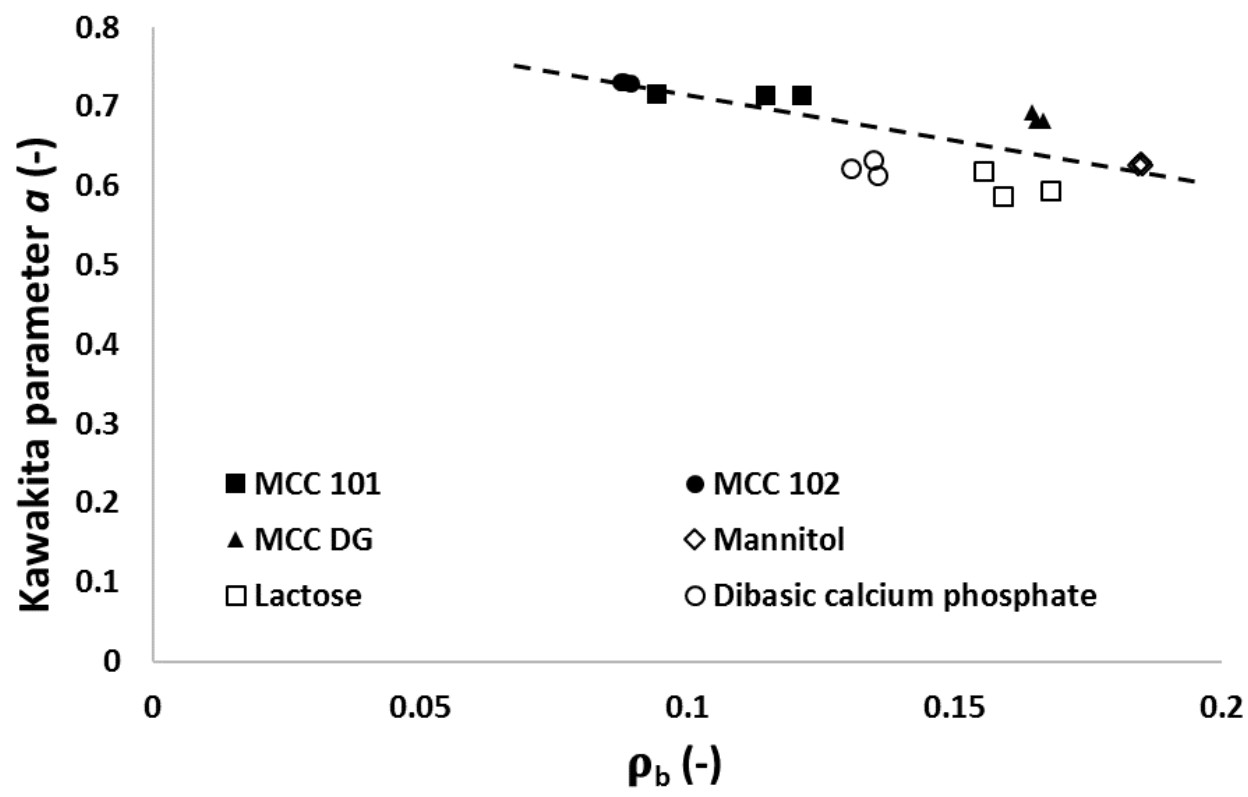

Figure 12. The relationship between the particle breakage (i.e., the breakage parameter $\rho_{\mathbf{b}}$ from Heckel analysis) and the powder compressibility (i.e., the Kawakita parameter a) with a compression pressure of $170 \mathrm{MPa}$.

The immediate elastic recovery of diluent powders had the similar negative relationship with the particle breakage (see Figure 13). It was shown that the immediate elastic recovery during the decompression process was related to the significance of particle fragmentation during compression. For MCC particles, a lesser extent of particle fragmentation (i.e., more particle deformation) was observed during powder compression process. Hence, MCC powders exhibited more significance of elastic deformation during decompression as the particle elastic deformation was positively related to the overall particle deformation. However, particles of mannitol, lactose, and DCP powders exhibited more particle fragmentation because the particles were more fragile and tended to be broken. As the particles in these powders broke into fragments and did not deform as much as MCC powders, relatively small elastic recovery was observed during the decompression process.

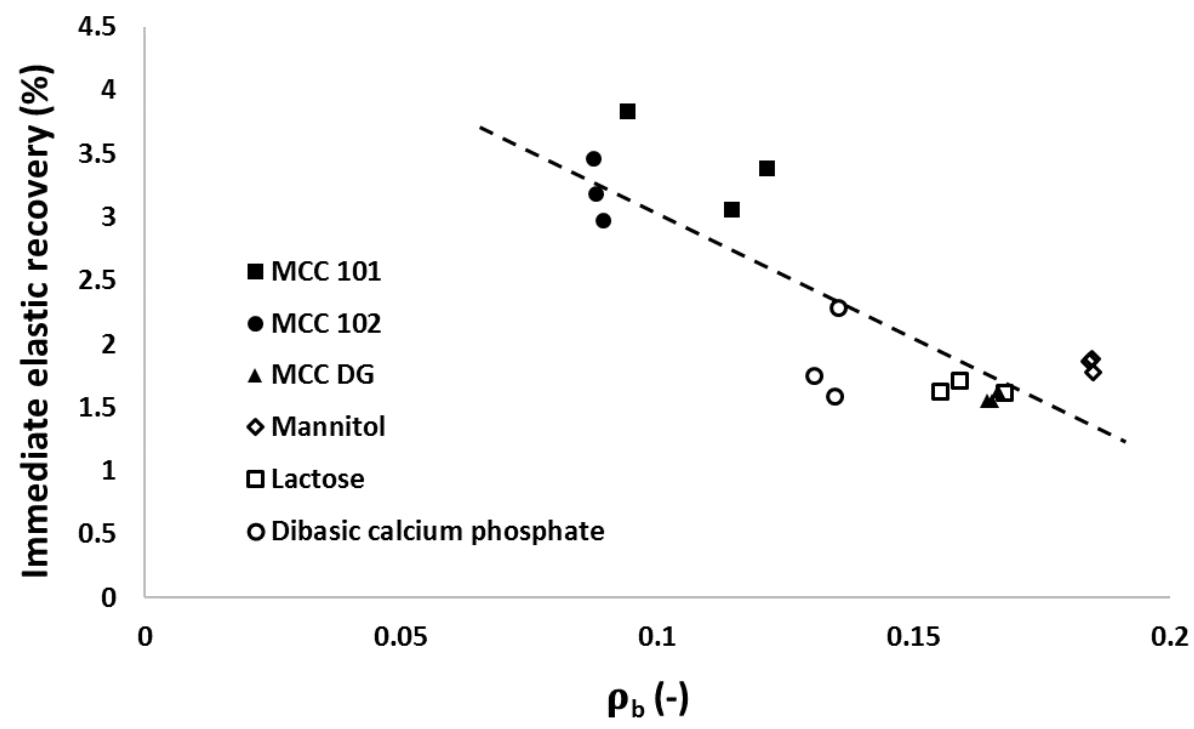

Figure 13. The relationship between the particle breakage (i.e., the breakage parameter $\rho_{\mathbf{b}}$ from Heckel analysis) and the in-die elastic recovery for different powders at a compression pressure of $170 \mathrm{MPa}$. 


\section{Conclusions}

In this paper, the properties of commonly-used pharmaceutical diluent powders were characterised. The compressibility was evaluated using both Heckel and Kawakita equations. The magnitude of particle rearrangement was explored using the Heckel analysis, considering both particle movement and particle fragmentation in the compression. The volume of the compressed tablet during the decompression process and in storage were monitored to determine the immediate elastic recovery (IER) and short-term elastic recovery (SER). Finally, the water absorbing ability of the powders was characterised. It was shown that, for all powder considered, they can be classified into two main categories according to the primary consolidation mechanism involved during compression:

(a) MCC PH 101, MCC PH 102, and MCC DG powders were characterised as the plastic materials because the particle deformation contributed significantly to powder volume reduction during compression. The plastic materials showed relatively good compressibility. At the beginning of the compression, the particles tended to relocate in order to reach the higher solid fraction. The powder also had good hygroscopicity and more water was absorbed when its surrounding relative humidity increased.

(b) Mannitol SD 100, lactose monohydrate, and dibasic calcium phosphate powders were brittle materials because their particles were more fragile compared with plastic materials. Since the powder was mainly consolidated by particle fragmentation, the compressibility of brittle materials was not as good as plastic materials. The magnitude of particle movement and particle fragmentation were affected by particle shape and individual particle strength. For the tablets made of mannitol powder, the volume exhibited shrinkage behaviours during storage. Furthermore, the water absorbed from the environment by the brittle powder was not sensitive to the storage relative humidity (i.e., from $10 \%$ to $85 \%$ ).

The negative relationships between the significance of particle breakage during compression and material bulk properties (i.e., compressibility and in-die elasticity) were also discovered. Mannitol, lactose, and DCP powders had more significance of particle breakage than MCC powders during compression, which contributed to the poorer compressibility and lower in-die elastic recovery for those powders. This implies that the significance of particle breakage is a critical material attribute that can be well characterised experimentally.

Author Contributions: J.Z. and C.Y.W. designed the experiments; J.Z. performed the experiments and drafted the manuscript; J.Z., C.Y.W., X.P. and C.W. analysed the data; C.Y.W., X.P. and C.W. revised the paper.

Conflicts of Interest: The authors declare no conflicts of interest.

\section{References}

1. Sastry, S.; Nyshadham, J.; Fix, J. Recent technological advances in oral drug delivery-A review. Pharm. Sci. Technol. Today 2000, 3, 138-145. [CrossRef]

2. Li, K.; Robinson, R. Influence of drug properties and routes of drug administration on the design of sustained and controlled release systems. In Controlled Drug Delivery_Fundamentals and Applications; Marcel Dekker: New York, NY, USA, 1987; pp. 3-94.

3. Overview of Pharmaceutical Excipients Used in Tablets and Capsules. Drug Topics, 24-10-2008. Available online: http://drugtopics.modernmedicine.com/drug-topics/news/modernmedicine/modern-medicinenews/overview-pharmaceutical-excipients-used-tablets (accessed on 10 March 2017).

4. Introduction to Tableting by Direct Compression. Available online: http://www.dfepharma.com/en/ knowledge-base/oral-solid-dose/direct-compression.aspx (accessed on 10 March 2017).

5. Narayan, P.; Hancock, B.C. The relationship between the particle properties, mechanical behaviour and surface roughness of some pharmaceutical excipient compacts. Mater. Sci. Eng. 2003, A355, 24-36. [CrossRef]

6. Akseli, I.; Hancock, B.; Cetinkaya, C. Non-destructive determination of anisotropic mechanical properties of pharmaceutical solid dosage forms. Int. J. Pharm. 2009, 377, 35-44. [CrossRef] [PubMed] 
7. Dave, V.S.; Fahmy, R.M.; Hoag, S.W. Investigation of the physical-mechanical properties of Eudragit RS, PO/RL, PO and their mixtures with common pharmaceutical excipients. Drug Dev. Ind. Pharm. 2013, 39, 1113-1125. [CrossRef] [PubMed]

8. Chitu, T.; Oulahna, D.; Hemati, M. Rheology, granule growth and granule strength: Application to the wet granulation of lactose-MCC mixtures. Powder Technol. 2011, 208, 441-453. [CrossRef]

9. Sun, C.; Kleinebudde, P. Mini review: Mechanisms to the loss of tabletability by dry granulation. Eur. J. Pharm. Biopharm. 2016, 106, 9-14. [CrossRef] [PubMed]

10. Kottala, N.; Abebe, A.; Sprockel, O.; Bergum, J.; Nikfar, F.; Cuitino, A.M. Evaluation of the Performance Characteristics of Bilayer Tablets: Part I. Impact of Material Properties and Process Parameters on the Strength of Bilayer Tablets. AAPS PharmSciTech. 2012, 13, 1236-1242. [CrossRef] [PubMed]

11. Akseli, I.; Ladyzhynsky, N.; Kata, J.; He, X. Development of predictive tools to assess capping tendency of tablet formulations. Powder Technol. 2013, 236, 139-148. [CrossRef]

12. Salbu, L. Compressibility and Compactibility of Pectin Powders-A Study of Their Potential as Direct Compression Excipients in Tablets. Ph.D. Thesis, Department of Pharmacy, University of Tromso, Tromso, Norway, 2011.

13. Heckel, R. An analysis of powder compaction phenomena. Trans. Metall. Soc. AIME 1961, 221, 1001-1008.

14. Haware, R.V.; Tho, I.; Bauer-Brandl, A. Evaluation of a rapid approximation method for the elastic recovery of tablets. Powder Technol. 2010, 202, 71-77. [CrossRef]

15. Hoag, S.; Dave, V.; Moolchandani, V. Compression and compaction. In Pharmaceutical Dosage Forms: Tablets; Volume 1: Unit Operations and Mechanical Properties; Augsburger, L., Hoag, S., Eds.; CRC Press: Boca Raton, FL, USA, 2008; pp. 573-574.

16. Duberg, M.; Nystrom, C. Studies on direct compression of tablets XVII. Porosity-pressure curves for the characterization of volume reduction mechanisms in powder compression. Powder Technol. 1986, 46, 67-75. [CrossRef]

17. Armstrong, N.; Haines-Nutt, R. Elastic recovery and surface area changes in compacted powder systems. J. Pharm. Pharmacol. 1972, 24, 135-136. [CrossRef]

18. Maganti, L.; Celik, M. Compaction studies on pellets. I. Uncoated pellets. Int. J. Pharm. 1993, 95, $29-42$. [CrossRef]

19. Denny, P. Compaction equations: A compression of the Heckel and Kawakita equations. Powder Technol. 2002, 127, 162-172. [CrossRef]

20. Nokhodchi, A.; Ford, J.; Rowe, P.; Rubinstein, M. The effect of moisture on the Heckel and energy analysis of hydroxypropyl methylcellulose 2208 (HPMC K4M). J. Pharm. Pharmacol. 1996, 48, 1122-1127. [CrossRef] [PubMed]

21. Klevan, I. Compression Analysis of Pharmaceutical Powders: Assessment of Mechanical Properties and Tablet Manufacturability Prediction. Ph.D. Thesis, University of Tromso, Tromso, Norway, 2011.

22. Patel, S.; Kaushal, A.M.; Bansal, A.K. Mechanistic investigation on pressure dependency of Heckel parameter. Int. J. Pharm. 2010, 389, 66-73. [CrossRef] [PubMed]

23. Dumarey, M.; Wikstrom, H.; Fransson, M.; Sparen, A.; Tajarobi, P.; Josefson, M.; Trygg, J. Combining experimental design and orthogonal projections to latent. Int. J. Pharm. 2011, 416, 110-119. [CrossRef] [PubMed]

24. Inman, S.; Briscoe, B.; Pitt, K.; Shiu, C. Axial tensile fracture of microcrystalline cellulose compacts. Int. J. Pharm. 2008, 349, 172-179. [CrossRef] [PubMed]

25. Souihi, N.; Dumarey, M.; Wikstron, H.; Tajarobi, P.; Fransson, M.; Svensson, O.; Josefson, M.; Trygg, J. A quality by design approach to investigate the effect of mannitol and dicalcium. Int. J. Pharm. 2013, 447, 47-61. [CrossRef] [PubMed]

26. Busignies, V.; Leclerc, B.; Porion, P.; Evesque, P.; Couarraze, G.; Tchoreloff, P. Compaction behaviour and new predictive approach to the compressibility of binary mixtures of pharmaceutical excipients. Eur. J. Pharm. Biopharm. 2006, 64, 66-74. [CrossRef] [PubMed]

27. Ilija, I.; Kasa, P., Jr.; Dreu, R.; Pintye-Hodi, K.; Srcic, S. The compressibility and compactibility of different types of lactose. Drug Dev. Ind. Pharm. 2009, 10, 1271-1280.

28. Schmidt, P.; Herzog, R. Calcium phosphates in pharmaceutical tableting. Pharm. World Sci. 1993, 15, 116-122. [CrossRef] [PubMed] 
29. Abebe, A.; Akseli, I.; Sprockel, O.; Kottala, N.; Cuitino, A.M. Review of bilayer tablet technology. Int. J. Pharm. 2014, 461, 549-558. [CrossRef] [PubMed]

30. Antikainen, O.; Yliruusi, J. Determing the compression behaviour of pharmaceutical powders from the force-distance compression profile. Int. J. Pharm. 2003, 252, 253-261. [CrossRef]

31. Yu, A.; Hall, J. Packing of fine powders subjected to tapping. Powder Technol. 1994, 78, 247-256. [CrossRef]

32. Patel, S.; Kaushal, A.M.; Bansal, A.K. Effect of particle size and compression force on compaction behaviour and derived mathematical parameters of compressibility. Pharm. Res. 2007, 24, 111-124. [CrossRef] [PubMed]

33. Schiano, S. Dry Granulation Using Roll Compaction Process: Powder Characterization and Process Understanding. Ph.D. Thesis, University of Surrey, Guildford, UK, 2017.

34. Govedarica, B.; Ilic, I.; Sibanc, R.; Dreu, R.; Srcic, S. The use of single particle mechanical properties for predicting the compressibility of pharmaceutical materials. Powder Technol. 2012, 225, 43-51. [CrossRef]

35. Haware, R.V.; Tho, I.; Baure-Brandl, A. Application of multivariate methods to compression behaviour evaluation of directly compressible materials. Eur. J. Pharm. Biopharm. 2009, 72, 148-155. [CrossRef] [PubMed]

36. Kachrimanis, K.; Malamataris, S. Apparent Young's elastic modulus and radial recovery for some tableted pharmaceutical excipients. Eur. J. Pharm. Sci. 2004, 21, 197-207. [CrossRef] [PubMed]

37. Celik, M.; Travers, D.N. The use of an elastic recovery index as a criterion of compactional behaviour of some direct compression bases. Drug Dev. Ind. Pharm. 1985, 11, 299-314. [CrossRef]

38. Roberts, R.; Rowe, R.; York, P. The Poisson's ratio of microcrystalline cellulose. Int. J. Pharm. 1994, 105, 177-180. [CrossRef]

39. Yoshinari, T.; Forbes, R.; York, P.; Kawashima, Y. Moisture induced polymorphic transition of mannitol and its morphological transformation. Int. J. Pharm. 2002, 247, 69-77. [CrossRef]

(C) 2017 by the authors. Licensee MDPI, Basel, Switzerland. This article is an open access article distributed under the terms and conditions of the Creative Commons Attribution (CC BY) license (http:/ / creativecommons.org/licenses/by/4.0/). 